\title{
Demonstration of gaps due to Jupiter in meteoroid streams. What happened with the 2003 Pi-Puppids?
}

\author{
J. Vaubaillon and F. Colas

\begin{abstract}
Institut de Mécanique Céleste et de Calcul des Éphémérides, Observatoire de Paris, 77 avenue Denfert-Rochereau, 75014 Paris, France

e-mail: vaubaill@imcce.fr
\end{abstract} \\ Received 2 June 2004 / Accepted 16 August 2004

\begin{abstract}
We simulated the dynamics of the Pi-Puppid meteoroid stream. The evolution of such a short period stream is dominated by close encounters with Jupiter. The effect is so great that it can cause the stream to split into several parts and form gaps. The difference in period of each part leads to the possibility of a meteor outburst on Earth while the parent body is at aphelion. Past observations are linked to streams ejected in the 19th and 20th century. Sometimes there are overlaps between several streams ejected at different perihelion passages of the parent body, affecting the time of maximum meteor activity. Generally speaking, observations suffer from a lack of coverage. In the 1972 and 2003 cases, only radio observations are available, and they are not in accordance with our predictions. This can be due to the radio detectors' lack of sensivity or to the poor knowledge of the efficiency of this physical process.
\end{abstract}

Key words. meteors, meteoroids - celestial mechanics - comets: individual: 26P/Grigg-Skjelerupp

\section{Introduction}

The evolution of meteoroid streams has been simulated by several authors (McNaught \& Asher 1999; Brown \& Jones 1998; Lyytinen \& Van Flandern 2000; Vaubaillon 2002; Vaubaillon \& Colas 2002; Wu \& Williams 1995). These works deal with meteoroid streams associated with a Halley type comet (Leonids, Perseids) or a short period comet (Draconids). In the same way, we will hereafter call these streams "Halley type" or "short period" meteoroid streams (Horner et al. 2003). The purpose of this paper is to look at the evolution of a short period meteoroid stream, associated with a Jupiter family comet: the Pi-Puppids.

The Pi-Puppid meteor shower results from a change in the orbit of comet 26P/Grigg-Skjellerup due to a close encounter with Jupiter in 1964 (Sitarski 1981; Kresak 1987). The main effect of this orbit change was to move the ascending node of the comet closer to the Earth's orbit, and hence meteor showers were expected during each comet return. Subsequently, there was another encounter with Jupiter in 1999, following which the ascending node is now far away from the Earth's orbit with "a priori" no possible meteor shower.

However, Messenger (2002) predicted that the Earth would cross radio meteor clouds in April 2003. These clouds are composed of small particles (20 to 100 microns) which were ejected from 1967 to 1997 . Messenger used a simple (but fast) model to predict possible aerocapture of dust during the 2003 meteor shower. His study is the first step towards accurate Pi-Puppid forecasts, and has motivated this work. Our goal was to use our new method (Vaubaillon 2002, 2004) developed for
Leonid meteor storms in order to predict accurate times of all Pi-Puppid apparitions.

First we will describe our physical model adapted to $26 \mathrm{P} /$ Grigg-Skjellerup, and mention the differences to Messenger's approach. In Sect. 3 we discuss the behaviour of a short period meteoroid stream, and the appearance of gaps due to close encounters with planets. Then we link past Pi-Puppid observations with streams ejected at various perihelion passages of 26P since 1848. The 1972 and 2003 cases need further attention and are the subject of Sect. 5. We present in particular the 2003 observations.

\section{The model}

The model is based on numerical integration of the orbits of several thousands of particles ejected by the comet and has been described in Vaubaillon $(2002,2004)$; Vaubaillon et al. (2003). However, in this work, no Hourly Rate (ZHR, see Koschack \& Rendtel 1990) computation from photometric observations of the parent body is carried out, as the observed 2003 shower consisted of mostly radio meteors (i.e. there are no ZHR observations available to calibrate such computations). We list below the differences between Messenger's (Messenger 2002) model and our method:

- Heliocentric distances of ejection are below 3 au, since water is the major component of comets, and begins to sublimate at this point (Messenger considered the whole orbit).

- The ejection velocity is computed based on a complete hydrodynamical model developed by Crifo \& Rodionov (1997). The radius of the cometary nucleus is $1.5 \mathrm{~km}$ 
(Boehnhardt et al. 1999). This model gives generally lower values of ejection velocity than Messenger's. In both cases, dust particles are ejected in the sunlit hemisphere.

- The gravitational influence of the 8 planets (Mercury to Neptune) and the Moon, solar radiation and PoyntingRobertson drag forces (Burns et al. 1979; Olsson-Steel 1987) are taken into account (Messenger had Keplerian orbits and considered perturbation by radiative forces only).

- We considered 4 size bins: 50-100 microns, 0.1-0.5 mm, $0.5-1 \mathrm{~mm}$ and, $1-5 \mathrm{~mm}$. The radio and visual meteor ranges are then covered. Messenger considered only radio meteors $(20-100 \mu \mathrm{m})$. The density is assumed to be $2000 \mathrm{~kg} \mathrm{~m}^{-3}$. $10^{4}$ particles per size bin were simulated, for a total of $4 \times 10^{4}$ particles per perihelion return. Usually, a comet ejects more small particles than big ones. The size distribution can be modelled by a power law of index $2<s<4$. However, our method ensures that the larger particle sizes are modelled in sufficient detail. If desired, weights can be applied to the various size bins to allow for the true size distribution.

- Perihelion returns from 1848 to 2002 were taken into consideration. They were obtained from P. Rocher (personal communication). In total, $1.28 \times 10^{6}$ particles have been simulated, from 32 perihelion returns, compared to Messenger, who simulated $6 \times 10^{5}$ particles for 6 returns.

- Since the orbits are perturbed by the planets, a numerical integration is needed. The program (Fortran 90) was run for each size bin. In order to make these computations faster, we used 10 processors of an IBM SP at CINES (Centre Informatique National de l'Enseignement Supérieur, FRANCE).

- A space criterion $\Delta X=\Delta T . V_{\mathrm{e}}$ (see Vaubaillon 2002) has been applied so as to consider only those particles which reach their nodes close enough to the Earth. As the relative speed of the encounter is low $\left(18.5 \mathrm{~km} \mathrm{~s}^{-1}\right.$, Lindblad (1987)), we have chosen $\Delta T=20$ days, such that $\Delta X \simeq 0.2 \mathrm{au}$, exactly as for our prediction for the Leonids. Messenger did not define any space criterion.

In our opinion, the facts that Messenger considered the 2-body problem and did not define any space criterion mainly explain the differences with our results shown below.

\section{Dynamics of a short period meteoroid stream}

Several studies (Asher 2000, 1999; McNaught \& Asher 1999; Lyytinen \& Van Flandern 2000; Vaubaillon 2002) have shown that even if meteoroid streams are close to their parent body, their orbits are still slightly different from each other, due to ejection processes and non-gravitational forces. The first consequence is an extension of the stream along comet orbits. Close encounters with giant planets (especially Jupiter, in the case of the Pi-Puppids) will have a crucial effect on the evolution of the stream by producing gaps. Studies of the Leonids (McNaught \& Asher 2002; Vaubaillon 2002) have shown that, because of the differential gravitational perturbations along the stream, gaps could appear in its structure. This is the most important effect of close encounters, since, most often, the tidal forces of such encounters only increase the extent of a stream. For the Pi-Puppids, and in general for short period meteoroid streams, the role of Jupiter is dominant for two reasons. First, close encounters would be more frequent than for Halley type streams, and second, the encounter occurs at aphelion, where the relative speed is minimum. Hence the lifetime of such a stream is expected to be shorter than, for example, the Perseids, in terms of the number of revolutions. Close encounters with Pi-Puppid meteoroid streams occurred in 1881, 1940, 1964 and 1999, and for the duration considered in this paper, will also occur in 2023 and 2047. It appears that 2 streams with similar conditions of ejection (cometary elements) can have a very different behaviour. This is the case, for example, of the 1873 and 1878 streams. In 2003, they appear as shown in Figs. 1 and 2. This difference can be explained by the fact that the first close encounter between these streams and Jupiter was in 1881. As the 1873 stream is older than the 1878 stream, it was a bit more extended, and then, was more sensitive to the encounter, since some parts of it were closer to the planet. Its extent was then increased, whereas the 1878 stream stayed confined. Thereafter, during every following close encounter with Jupiter, since the 1873 stream is more extended, it was more likely to be perturbed than the 1878 stream.

Another interesting feature seen in Fig. 1 is that, due to the repeated perturbations and disruptions of the stream, a "double stream" can appear. The two components of the double stream can consist of different amounts of material. For example in Fig. 1, the outer component has more particles than the inner one.

A consequence of the disruption process is that, as can be seen in Fig. 1, the density of the parent stream varies along the orbit path and gaps can be created. We can recognize two distinct parts in this stream: the "head", close to the comet (approximate coordinates: $[1.0 ;-1.5]$ ), and the "dense tail" $[2.0 ; 3.0]$. The separation between these two dense parts of the stream can reach $180 \mathrm{deg}$ in true anomaly. It follows that a meteor outburst can be caused by the "tail" of the stream when the comet is at aphelion, as shown in Fig. 3.

Figure 5 shows a consequence of the existence of a double stream: the Earth's neighbourhood is filled with meteoroids from different components of the same stream. Though it is not the case in Fig. 5, it can be said that, generally, a stream from a single perihelion passage of the parent comet can cause multiple peaks during a meteor shower. After a sufficiently long time, this spreading process will also lower the level of each observed maximum, in such a way that no real maximum will finally be observable, as was the case in 1972, for example.

Figure 4 shows the expected high efficiency of the 2047 close encounter in dispersing the Pi-Puppids, and it can be seen that the 1888 stream is highly disturbed by repeated encounters. The ring shape is present in almost all streams simulated here.

\section{Connection between streams and past observations}

The main showers took place in 1972, 1977 and 1982 (see Baggaley 1973; Shao et al. 1977; Lindblad 1987; Hughes 1992), but it was impossible to explain them with the 


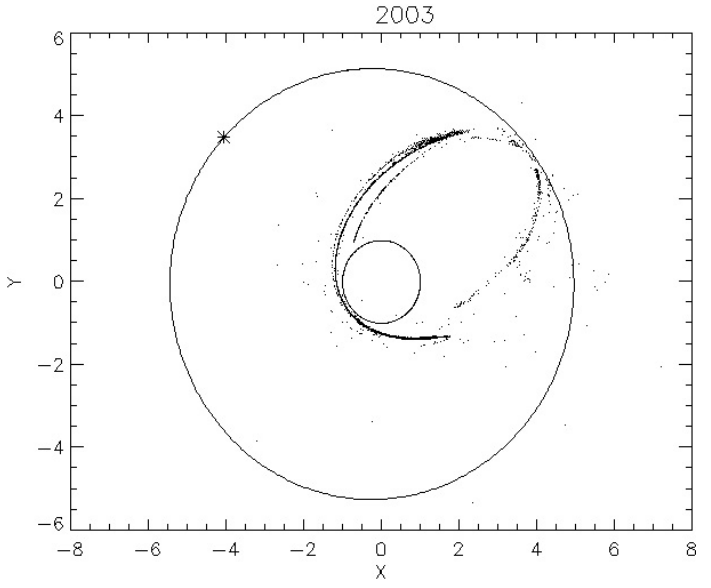

Fig. 1. 1873 stream as seen on 23rd Apr. 2003, in $(x, y)$ ecliptic J2000 coordinates. The big circle symbolises Jupiter's orbit, the asterisk the position of Jupiter, and the small circle the Earth's orbit. The particle sizes are in the bin $[0.1-0.5 \mathrm{~mm}]$.

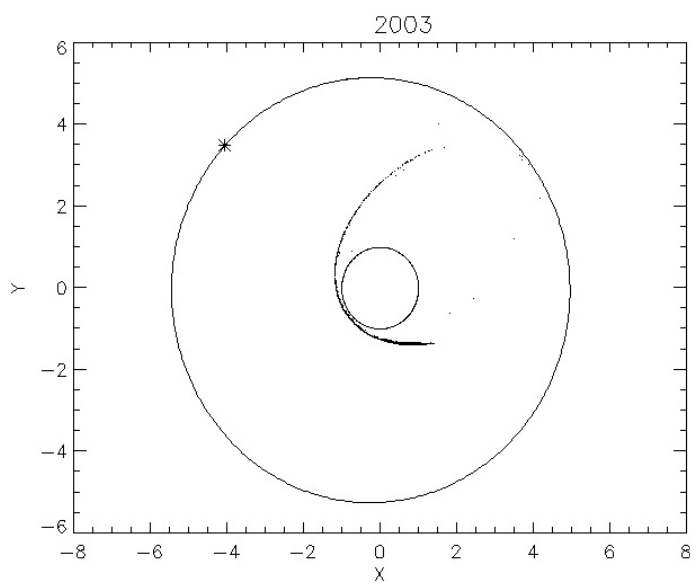

Fig. 2. 1878 stream as seen on 23rd Apr. 2003, in $(x, y)$ ecliptic J2000 coordinates. The particle sizes are in the bin $[0.1-0.5 \mathrm{~mm}]$.

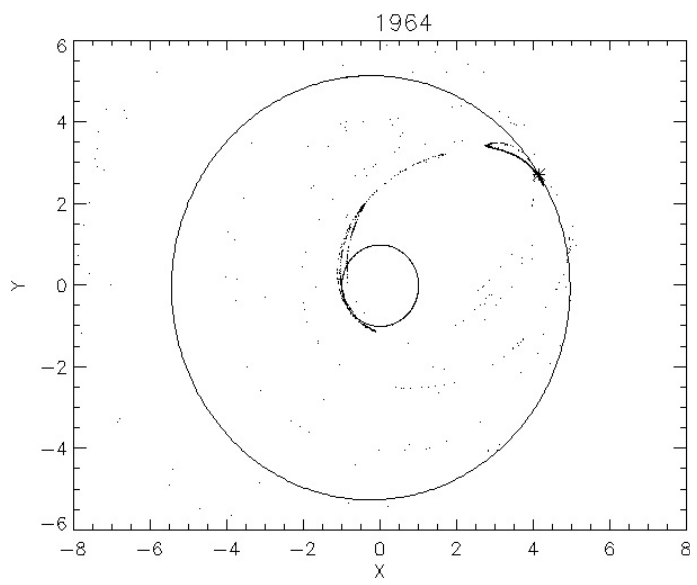

Fig. 3. 1888 stream, as seen in 1964. The comet is near aphelion, close to Jupiter, while there are many particles still at perihelion. It shows that meteor outbursts can happen even if the parent body is far from the orbit of the Earth. The particle sizes are in the bin $[0.1-0.5 \mathrm{~mm}$.

dust ejected during the 1967-1997 period, which is the time when the ascending node of the comet was closest to

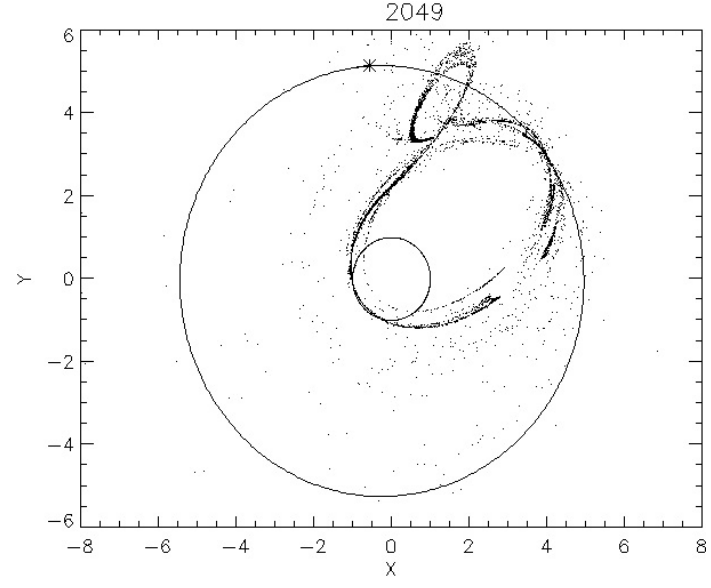

Fig. 4. 1888 stream, as seen in 2049. The ring shape is due to a close encounter with Jupiter in 2047, but the stream was already very perturbed by previous encounters, and shows multiple secondary streams. The particle sizes are in the bin [0.1-0.5 mm].

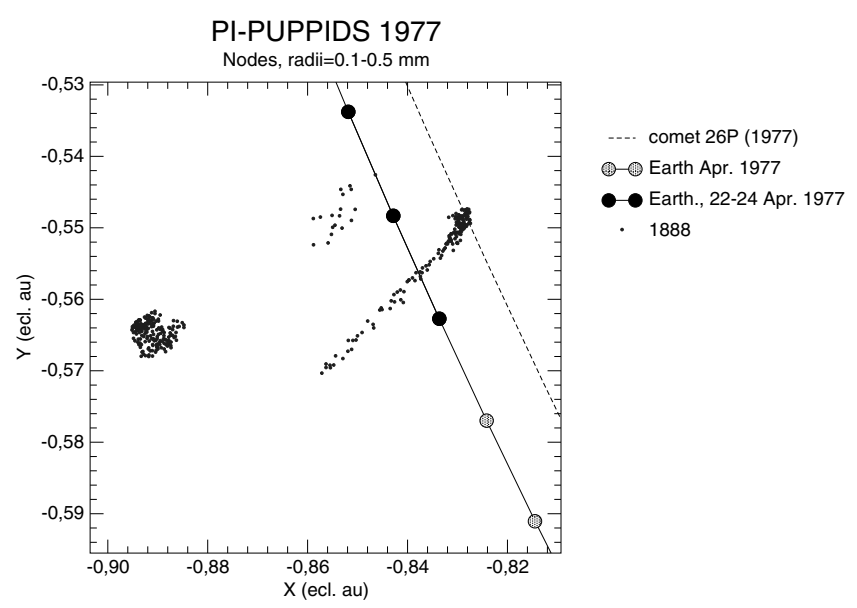

Fig. 5. Nodes of particles from the 1888 stream, in 1977. The projection of the comet orbit on the ecliptic is also shown.

Earth (Messenger 2002). Moreover, no Pi-Puppid meteor observations were reported in 1987, 1992 or 1997. Instead, Kondratueva \& Muravyova (1993) predicted that no meteor outburst was expected in 1997, based on the 1964 stream. Hence, we were surprised that we needed to consider old streams in order to explain the Pi-Puppids' behaviour (the oldest was the 1848 stream, as the comet orbit gets more unreliable at earlier times). We also had to carefully study the observations since errors can be significant due to the small ZHR and small speed of encounter (Lindblad 1987). Table 1 gives a summary of the observations (see also Hughes 1992), which are compared with our computations.

Comments relating to observations from individual years are as follows:

- 1977: Shao et al. (1977) noted a maximum around April 23.5-23.6 ( $\simeq 12: 00-14: 30$ UT). However, no shower was observed from Florida (IAU Circ. 3069, 3092) between 0:00 and 3:00 UT, suggesting that the shower was very short. The main contributors to this shower were the 1863 , 1868 and 1873 streams. If we consider only these streams, 
Table 1. Observations of Pi-Puppids and stream association. ${ }^{1}$ Observation method: $\mathrm{R}=$ radar, $\mathrm{V}=$ visible. ${ }^{2}$ Time (UT hour or decimal day) of observed Max., on April 23rd. ${ }^{3}$ Visible ZHR of the Maximum. ${ }^{4}$ Associated streams, in order of importance. $\mathrm{R}=$ radio meteors (50 microns$1 \mathrm{~mm}) ; \mathrm{V}=$ visible meteors $(\geq 1 \mathrm{~mm}){ }^{5}$ Time (UT hour or decimal day) of predicted Max. (on April 23rd, unless stated). ${ }^{6}$ Total duration of predicted shower. (see Hughes 1992, for a full review of observations).

\begin{tabular}{|c|c|c|c|c|c|c|c|}
\hline \multicolumn{4}{|c|}{ Observations } & \multicolumn{4}{|c|}{ Simulations } \\
\hline Year & Obs. $^{1}$ & Max. $(\mathrm{UT})^{2}$ & $\mathrm{ZHR}^{3}$ & Stream $^{4}$ & Max. $(\mathrm{UT})^{5}$ & Total $(h)^{6}$ & Comments \\
\hline 1972 & $\mathrm{R}$ & $\begin{array}{l}\text { no evident Maximum } \\
\text { Broad component }\end{array}$ & 2 & $\begin{array}{l}1967,1961(\mathrm{R})+ \\
1904\end{array}$ & $22 / 04,23: 30 \pm 30^{\prime}$ & 1 & see Sect. 5 \\
\hline 1977 & $\mathrm{~V}$ & $\begin{array}{l}23.5-23.6 \simeq 12: 00- \\
14: 30\end{array}$ & 75 & $\begin{array}{l}1873,1863,1868 \\
\text { and all from } 1848 \\
\text { to } 1907(\mathrm{~V})\end{array}$ & $13: 57 \pm 1: 00 \simeq 23.58$ & 10 & $\begin{array}{l}\text { Minor components } \\
\text { shift the Maximum } \\
\text { earlier }\end{array}$ \\
\hline 1982 & $\mathrm{~V}$ & $\begin{array}{l}\text { 11:00-12:00 but } \\
\text { probably earlier }\end{array}$ & 23 & $\begin{array}{l}1907(\mathrm{~V}) \text { and all } \\
\text { from } 1892 \text { to } 1922\end{array}$ & $\begin{array}{l}6: 00 \pm 1: 00 \text { or } 2 \text { peaks } \\
\text { at } 3: 00 \text { and } 9: 00\end{array}$ & 10 & $\begin{array}{l}\text { Missed Maximum: } \\
\text { only end visible }\end{array}$ \\
\hline 1987 & no shower & - & 0 & - & - & - & no outburst \\
\hline 1992 & idem & - & 0 & - & - & - & idem \\
\hline 1997 & idem & - & 0 & - & - & - & idem \\
\hline 2002 & idem & - & 0 & - & - & - & idem \\
\hline 2003 & $\mathrm{R}+\mathrm{V}$ & no evident Maximum & 0 & 1957, $1961(\mathrm{R})$ & $15: 00 \pm 1: 00$ & 7 & $\begin{array}{l}\text { single peak, see } \\
\text { Sect. } 5\end{array}$ \\
\hline
\end{tabular}

the maximum is found to occur later than the time given in Table 1. However, the overall effect of all other minor contributions from other streams is to shift the maximum to April 23.58 as shown in Table 1, which is a good match to the observations. This feature is interesting, since Hughes (1992) had mentioned that "overlap will occur". That means that a single meteor shower results from the encounter between the Earth and several different streams ejected at several perihelion returns, at a single given time. But this overlap between the 1873, 1863, 1868 and all other streams ejected between 1848 and 1902 is more or less efficient. Indeed, the meteor activity is wider than one resulting from a single stream only. This means that only a small part of a stream overlaps with another small part of a different stream. This kind of overlap has also been predicted and observed in the 2001 Leonid shower (Asher 2000; Arlt et al. 2001). Wood (cited by Hughes 1992) and Lindblad (1987) concluded from observations that the shower lasted for only a few hours. Our results, on the contrary, suggest a long shower $(10 \mathrm{~h})$. This longer duration means, though, that the shower began around 4:00 (UT). In such a case, nothing would have been seen in Florida between 0:00 and 3:00, as reported (IAU Circ. 3069, 3092). Lindblad (1987) also concluded that the correct ZHR could be 1000-4000 meteors rather than 75 as reported by Shao et al. (1977). The difference between these observations and estimation of $Z H R$ is caused by the extremely low velocity of the meteors, making lots of meteor fainter than if they were faster, and thus hard to detect. This effect, combined with the inefficient overlap can explain the fact that the shower seemed to be much shorter than suggested by our results.

- 1982: our results show that the maximum was missed, due to poor observation coverage (Australia only, see Hughes 1992). From his own analysis of the shower, Wood (cited by Hughes 1992) mentioned that the peak could be earlier than what was observed and indeed, this is what is found here. As in the 1977 case, we find that inefficient overlap occurs. This makes it hard to define a maximum and that is why one or two peaks are predicted. Overall we found a large meteor shower duration.

- 1987 to 1997: no meteor shower has been reported to our knowledge. Also no meteoroid stream was found to be close to the Earth during this period.

\section{The 1972 and 2003 cases}

These two years both have a radio outburst predicted. Figures 6 and 7 show the node of the particles selected by our space criterion (see Sect. 2)

1972: Baggaley (1973, Fig. 1) shows a broad component of the shower, lasting four days. We note that the observation on the 23rd April began after 0:00 UT (UT = NZST-12 hrs), and that the level of the shower was decreasing, but low. Our results suggest that a maximum occurred shortly prior to these observations, but the end of the shower should have been observable. The limiting magnitude given by the instrument was +8.5 .

2003: our results predicted an encounter with two radiometeoroid streams, ejected in 1957 and 1961. An encounter in 2003 was already predicted by Messenger (2002), but the streams involved are different here. He considered only the streams from the 1967-1997 period, and Keplerian orbits. He also did not define any space criterion, so that there is no information about the timing at which the particles that are considered as impacting reach their node. We have found here that the two streams overlap, whereas Messenger predicted two distinct maxima. Following these results, we made a call to observers on meteor observer mailing-lists (IMO-news and meteorobs). The observations are summarized in Table 2.

The fact that very few visual meteors have been observed is not surprising, since our prediction concerned only radio meteors. Generally speaking, we can say that observers were either not so well located (Northern hemisphere or far east part of the world), or had no suitable instruments (a radio station). The 
Table 2. Observations of 2003 Pi-Puppids, from IMO and meteorobs observation reports, and personal communications. We first report visual observations, then radio ones (forward scattering stations). Note that Hans Salm also did binocular observations, with the same result as reported here.

\begin{tabular}{|c|c|c|c|c|}
\hline Observer & Location & Method & Time (UT) & Results \\
\hline Hans Salm & Bolivia & visual & $23 / 043: 30-5: 30+24 / 043: 45-5: 15$ & no PPU \\
\hline Tim Cooper & South Africa & visual & $\begin{array}{l}23 / 04 \text { 18:46-19:55 +23/04 20:16- } \\
20: 37\end{array}$ & no PPU \\
\hline Mike Begbie & Zimbabwe & visual & 23/04 16:45-19:00 & 9 PPU between 17:05 and 17:15 \\
\hline $\begin{array}{l}\text { Josep Trigo-Rodríguez, Carles } \\
\text { Pineda, Albert Sánchez (Spanish } \\
\text { Fireball Network) }\end{array}$ & Spain & visual & $23 / 04$ & no PPU \\
\hline Yk Chia & Singapore & visual & $23 / 04 \quad 7: 00$ & no PPU (+ clouds) \\
\hline Quanzhi Ye & China & visual & $23 / 04$ & no PPU (+ clouds) \\
\hline Kazuhiro Osada & Japan & visual & $23 / 04$ & $1 \mathrm{PPU}$ \\
\hline Adam Marsh & Australia & visual & $22 / 04$ evening $+23 / 04$ morning & 4 PPU in 2 hours \\
\hline Michael Boschat & Canada & radio & $23 / 04$ 10:00-15:00 & Nothing special about PPU \\
\hline Jean-Marie Polard & Belgium & radio & $\begin{array}{llll}23 / 04 & 12: 56-24: 00 & 24 / 04 & 0: 00- \\
12: 56 & & & \\
\end{array}$ & Max. at 8:30 and 17:10 UT \\
\hline Marcel Schneider & Luxembourg & radio & $23 / 04$ & $\begin{array}{l}\text { Clear enhanced activity from } 13 \text { to } \\
17: 00\end{array}$ \\
\hline George Lauffer & Germany & radio & 23/04 0:00-24:00 & $\begin{array}{l}\text { Many echoes on the morning } \\
(7: 00)\end{array}$ \\
\hline Javor Kac, Jure Zakrajsek & Slovenia & radio & 23/04 16:00-20:00 & Sporadic E \\
\hline Bruce Young & Australia & radio & $22 / 04$ to $24 / 04$ & Nothing special about PPU \\
\hline $\begin{array}{l}\text { Hiroshi Ogawa (+ Nippon Meteor } \\
\text { Society and Japanese Radio } \\
\text { Observers) }\end{array}$ & Japan & radio & $23 / 04-24 / 04$ & $\begin{array}{l}\text { PPU activity observed, but no spe- } \\
\text { cial outburst on } 23 / 04\end{array}$ \\
\hline
\end{tabular}

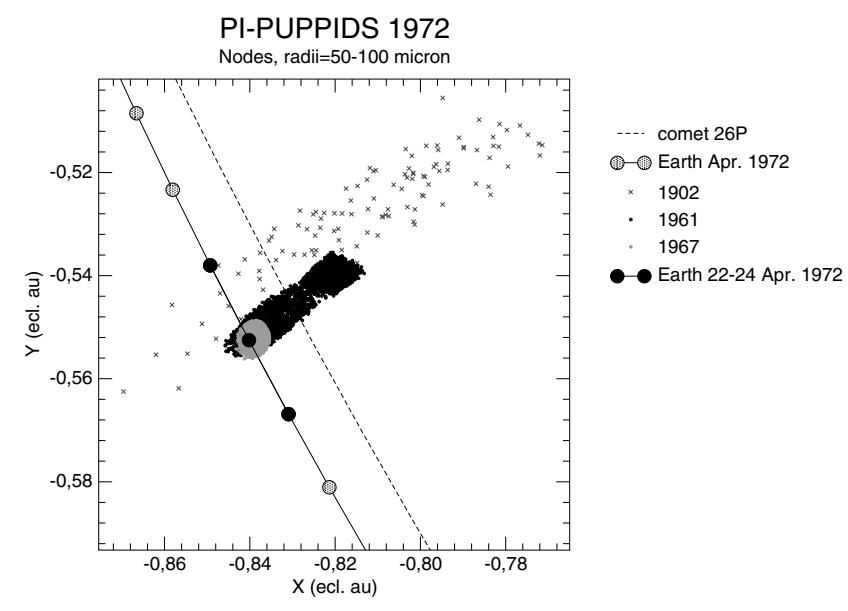

Fig. 6. Nodes of particles ejected in 1902, 1961 and 1967, intersecting the Earth's orbit in 1972.

best located European radio station was in Slovenia, but suffered from high sporadic E activity (J. Kac, personnal communication). The New Zealand radar was not operating the date of the shower (J. Baggaley, personal communication).

Only one radio station (forward scattering) observed activity in good agreement with our prediction (M. Schneider, Luxembourg). But there is no confirmation from neighbouring countries (Belgium, Germany); the results are not homogeneous. We regret that there were no observation from Antarctica, but we do not know if there is a radio meteor station in this part of the world. According to the predicted duration of the encounter, far east regions such as Japan or Australia could

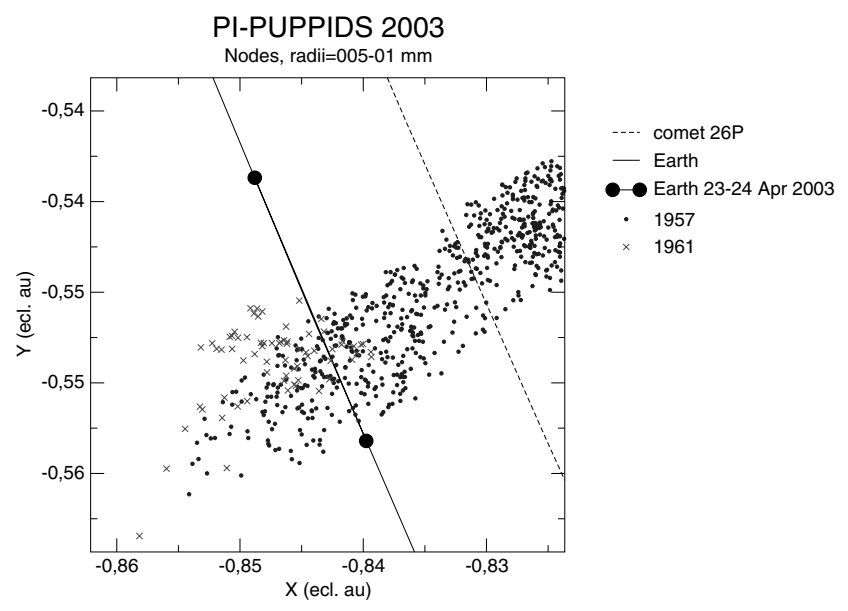

Fig. 7. Nodes of particles intersecting the Earth's orbit in 2003.

observe the end of the shower, but no outburst was reported, though a general Pi-Puppid activity was observed (Ogawa H.).

Looking at these results, we cannot say that a shower was observed as predicted.

Furthermore, we can remark that in both the 1972 and 2003 cases, the predicted or post-predicted radio-outbursts have not been confirmed. We now have two hypotheses to explain this. The first possibility is that there were no radio outbursts, and then, we must conclude that our model, that predicted the 2002 Leonids so well (Vaubaillon 2002; Arlt et al. 2002), cannot be applied to radio meteor particles. This would mean that small particles suffer other forces that we have not taken into account here (e.g. ionization process, see Gustafson 1994). 
The radiative effects taken into account here are solar radiation pressure and Poynting-Robertson drag. Note that the seasonal Yarkovsky effect taken into account by Lyytinen \& Van Flandern (2000) requires a difference of temperature over the meteoroid, that small particles would not likeky show. Olsson-Steel (1987) has indeed calculated that $100 \mu \mathrm{m}$ would be the smallest size of a particle to present this phenomenon.

The other possibility is that there was an encounter, but it could not be detected. The limiting magnitude of the radar used in 1972 by Baggaley (1973) is +8.5 . Figure 16 of Brown et al. (2000) provides the limiting magnitude of several techniques. It appears that the smallest detectable particle by a radio station is $10^{-9} \mathrm{~kg}$. Converting this mass into magnitude, according to Hughes (1995), one finds Mag $=+11$ (for an atmospheric entry speed $V_{\mathrm{e}}$ of $71 \mathrm{~km} \mathrm{~s}^{-1}$ used by Brown et al. (2000) for the Leonid case). Baggaley (1995) noted that the AMOR system used in 1995 had a limiting magnitude of +13 , corresponding to a meteoroid size of $\simeq 100 \mu \mathrm{m}$, (he used $V_{\mathrm{e}}=40 \mathrm{~km} \mathrm{~s}^{-1}$ ). Applying again Hughes' (1995) formula to the Pi-Puppids $\left(V_{\mathrm{e}}=18.5 \mathrm{~km} \mathrm{~s}^{-1}\right)$, one finds a magnitude of +14 , for such size range particles. This is fainter than can be detected by current radio meteor devices. Not all radio forward scattering stations have the same accuracy, so it is hard to define a limiting magnitude. But we can assume that this value will not exceed that of professional systems such as AMOR. A common value for amateur equipment is a limiting magnitude of $\simeq 6-8$, and rarely more than 12. A further analysis of Fig. 6 shows that particles encountered are restricted to the 50-80 micron size range (corresponding to meteors of magnitude $>+15$ ). Moreover, the entry speed of Pi-Puppids is the smallest among all meteor showers listed in the working list of the International Meteor Organization (see http://imo.net): $18.5 \mathrm{~km} \mathrm{~s}^{-1}$ (Lindblad 1987). This value is half that used by Baggaley (1995) to derive the lower limit of meteoroid size the AMOR system was able to detect in 1995. This low velocity is the reason why Messenger (2002) predicted a possible collection of micro-meteors. In this case, such a meteoroid (small and slow) is hard to detect, and this could explain why no special outburst has been observed. The positive observation done in Luxembourg could be an artifact, probably due to electro-magnetic activity in the atmosphere around April 23rd.

\section{Conclusion}

The evolution of a short period meteoroid stream is dominated by close encounters with Jupiter. This encounter causes some disruptions in the stream, following which, multiple streams could be created from a single pre-existing one. This makes the evolution so "complicated" (Hughes 1992) that a meteor outburst can occur even if the parent body is at aphelion.

Pi-Puppid meteor showers are generally long (several hours) and have a relatively low level. This is the result of the large activity dispersion of streams perturbed by Jupiter. The observed 1977 outburst has been shown to mainly result from material ejected in 1873, 1863 and 1868.
The fact that 1972 and 2003 observations are not in agreement with our simulations is due to the radio receptors' lack of sensivity. The modeling of radio meteors is also not well known. No other Pi-Puppid (visual or radio) outburst is expected in the coming years, at least until 2050.

Acknowledgements. We are grateful to CINES for providing computation support, J. F. Crifo for the cometary model, and S. Messenger and E. Lyytinen for discussions. We heartily thank all the observers for having shared their observations with us. This work is supported by CNES (French space agency).

\section{References}

Arlt, R., Kac, J., Krumov, V., Buchmann, A., \& Verbert, J. 2001, WGN, J. Int. Meteor Organization, 29, 187

Arlt, R., Krumov, V., Buchmann, A., Kac, J., \& Verbert, J. 2002, WGN, J. Int. Meteor Organization, 30, 205

Asher, D. J. 1999, MNRAS, 307, 919

Asher, D. J. 2000, in Proc. of the International Meteor Conference, Frasso Sabino, Italy, 23-26 September 1999, 5

Baggaley, W. J. 1973, The Observatory, 93, 23

Baggaley, W. J. 1995, Earth Moon and Planets, 68, 127

Boehnhardt, H., Rainer, N., Birkle, K., \& Schwehm, G. 1999, A\&A, 341,912

Brown, P., \& Jones, J. 1998, Icarus, 133, 36

Brown, P., Campbell, M. D., Ellis, K. J., et al. 2000, Earth Moon and Planets, 82, 167

Burns, J. A., Lamy, P. L., \& Soter, S. 1979, Icarus, 40, 1

Crifo, J. F., \& Rodionov, A. V. 1997, Icarus, 127, 319

Gustafson, B. A. S. 1994, Ann. Rev. Earth Planetary Sci., 22, 553

Horner, J., Evans, N. W., Bailey, M. E., \& Asher, D. J. 2003, MNRAS, 343,1057

Hughes, D. W. 1992, MNRAS, 257, 25P

Hughes, D. W. 1995, Earth Moon and Planets, 68, 31

Kondratueva, E. D., \& Muravyova, I. N. 1993, Astronomicheskii Vestnik, 27, 120

Koschack, R., \& Rendtel, J. 1990, WGN, J. Int. Meteor Organization, 18,119

Kresak, L. 1987, Bull. Astron. Inst. Czechoslovakia, 38, 65

Lindblad, B. A. 1987, A\&A, 187, 931

Lyytinen, E. J., \& Van Flandern, T. 2000, Earth Moon and Planets, 82, 149

McNaught, R. H., \& Asher, D. J. 1999, WGN, J. Int. Meteor Organization, 27, 85

McNaught, R. H., \& Asher, D. J. 2002, WGN, J. Int. Meteor Organization, 30, 132

Messenger, S. 2002, Meteoritics and Planetary Science, 37, 1491

Olsson-Steel, D. 1987, MNRAS, 226, 1

Shao, C.-Y., Bulger, J. H., Suzuki, K., et al. 1977, IAUC, 3092, 4

Sitarski, G. 1981, Acta Astron., 31, 479

Vaubaillon, J. 2002, WGN, J. Int. Meteor Organization, 30, 144

Vaubaillon, J. 2004, Ph.D. Thesis, Observatoire de Paris

Vaubaillon, J., \& Colas, F. 2002, Proc. of Asteroids, Comets, Meteors - ACM 2002, International Conference, 29 July-2 August 2002, Berlin, Germany, ed. B. Warmbein, ESA SP-500 (Noordwijk, Netherlands: ESA Publications Division), 181

Vaubaillon, J., Lyytinen, E., Nissinen, M., \& Asher, D. 2003, WGN, J. Int. Meteor Organization, 31, 131

Wu, Z., \& Williams, I. P. 1995, P\&SS, 43, 723 